1990-01-01

\title{
Discussion of the representation of intercrystalline misorientation in cubic materials
}

\author{
Brent L. Adams \\ b_I_adams@byu.edu \\ Hans Grimmer \\ Junwu Zhao
}

Follow this and additional works at: https://scholarsarchive.byu.edu/facpub

Part of the Mechanical Engineering Commons

Original Publication Citation

Acta Cryst. (199). A46, 62-622

\section{BYU ScholarsArchive Citation}

Adams, Brent L.; Grimmer, Hans; and Zhao, Junwu, "Discussion of the representation of intercrystalline misorientation in cubic materials" (1990). Faculty Publications. 734.

https://scholarsarchive.byu.edu/facpub/734

This Peer-Reviewed Article is brought to you for free and open access by BYU ScholarsArchive. It has been accepted for inclusion in Faculty Publications by an authorized administrator of BYU ScholarsArchive. For more information, please contact ellen_amatangelo@byu.edu. 
Discussion of the representation of intercrystalline misorientation in cubic materials. By BRENT L. AdAMS and Junwu ZhaO, Department of Mechanical Engineering, Yale University, New Haven, CT 06520-2157, USA and HANS GRIMMER, Paul Scherrer Institute, Laboratory of Materials Science, CH-5232 Villigen PSI, Switzerland

\begin{abstract}
Salient features of various parameterizations of cubic-cubic misorientation are discussed. It is proposed that the quaternion representation of rotations, as a pair of antipodal points on the surface of a four-dimensional sphere, encompasses the most desirable properties of other proposed representations, viz rectilinearity, a closed form for the composition of successive rotations, and an equivalence between the Euclidean measure on its parameter space and the invariant measure in the space of rotations. The classification of cubic-cubic misorientations according to group multiplicity is described in Euler angle and quaternion representations. A correspondence between coincidence site lattice (CSL) boundaries $(\Sigma \leq 49)$, Euler angles and axis-angle parameters is given.
\end{abstract}

The following pertains to the recent paper of Zhao \& Adams (1988), entitled Definition of an Asymmetric Domain for Intercrystalline Misorientation in Cubic Materials in the Space of Euler Angles, and subsequent comments of Grimmer (1989). It is clear that the Euler angle representation of misorientation suffers from a number of disadvantages as discussed by Altmann (1986), Frank (1988), Grimmer (1989), and others. However, quantitative descriptions of orientation and misorientation distribution functions have usually been expressed in Fourier series using generalized spherical harmonics (Bunge, 1982); and these are defined in terms of Euler angles (Gelfand, Minlos \& Shapiro, 1963). In their calculation of the misorientation distribution function (MDF) in copper, for example, Pospiech, Sztwiertnia \& Haessner (1986) used the space of Euler angles for computation, and later transformed to the axis-angle parameters. Comparable orthogonal basis functions for axis-angle, quaternion, Rodrigues or other parameterizations have not yet been defined, even though they would be valuable. The work of Zhao \& Adams (1988) was motivated by the pressing need to represent continuous functions, in the smallest physically distinctive domain of cubic-cubic misorientation, given the necessity of using Euler angles. The definition of an asymmetric domain significantly reduces computation time and increases the clarity of representation.

The quaternion representation described in the comments by Grimmer has some significant advantages. This representation, due to Handscomb (1958), defines rotation by a pair of antipodal points on the hypersurface of a unit sphere in four-dimensional space. [Note that this is not the quaternion parameter $Q$ of Frank (1988), which is obtained from Handscomb's quaternion by omitting its fourth component.] Handscomb shows in his concise paper that his representation has the following properties. It has the rectilinearity property of Frank's mapping (ii). In fact Handscomb obtains the semi-regular truncated cube by considering the quaternions corresponding to minimum angle descriptions of misorientations between cubic crystals. It also has the property that the result of two successive rotations can be calculated as easily as in Frank's mapping (iii). Finally it has the property that the Euclidean measure on its parameter space corresponds to an invariant measure in the space of rotations as in Frank's mapping (iv). In summary, it combines the advantages of Frank's mappings (ii)-(iv) at the price of using four dimensions instead of three. Conversely, the price of going to three dimensions is that at most one of the three desired properties can be maintained.

Table 2 of the previous paper by Zhao \& Adams contains some errors as noted by Grimmer. Table 1 of this comment is a corrected table. It is correct that only boundaries with rotation axis $[1,1,1]$ should be classified as $m=6$. This statement is in good agreement with the analysis presented in section 3 of the paper (Zhao \& Adams, 1988). Boundaries 
Table 1. CSL boundaries for $\Sigma \leq 49$ ( $m$ is the multiplicity)

\begin{tabular}{|c|c|c|c|c|c|c|c|c|c|c|c|c|c|}
\hline \multirow[b]{2}{*}{$\Sigma$} & \multirow[b]{2}{*}{$m$} & \multicolumn{3}{|c|}{ Euler angles } & \multicolumn{2}{|c|}{ Axis-angle } & \multirow[b]{2}{*}{$\Sigma$} & \multirow[b]{2}{*}{$m$} & \multicolumn{3}{|c|}{ Euler angles } & \multicolumn{2}{|c|}{ Axis-angle } \\
\hline & & $\varphi_{1}$ & $\phi$ & $\varphi_{2}$ & $\langle h, k, l\rangle$ & $\omega$ & & & $\varphi_{1}$ & $\phi$ & $\varphi_{2}$ & $\langle h, k, l\rangle$ & $\omega$ \\
\hline 3 & 12 & $45 \cdot 00$ & $70 \cdot 53$ & $45 \cdot 00$ & $1,1,1$ & $60 \cdot 00$ & $33 b$ & 2 & $12 \cdot 34$ & 83.04 & $58 \cdot 73$ & $3,1,1$ & $33 \cdot 56$ \\
\hline 5 & 8 & 0.00 & $90 \cdot 00$ & $36 \cdot 86$ & $1,0,0$ & $36 \cdot 86$ & $33 c$ & 4 & $38 \cdot 66$ & 75.97 & $38 \cdot 66$ & $1,1,0$ & 58.99 \\
\hline 7 & 6 & $26 \cdot 56$ & $73 \cdot 40$ & 63.44 & $1,1,1$ & $38 \cdot 21$ & $35 a$ & 2 & $16 \cdot 86$ & $80 \cdot 13$ & $60 \cdot 46$ & $2,1,1$ & $34 \cdot 05$ \\
\hline 9 & 4 & $26 \cdot 56$ & $83 \cdot 62$ & $26 \cdot 56$ & $1,1,0$ & $38 \cdot 94$ & $35 b$ & 2 & 30.96 & $88 \cdot 36$ & $59 \cdot 04$ & $3,3,1$ & $43 \cdot 23$ \\
\hline 11 & 4 & $33 \cdot 68$ & $79 \cdot 53$ & $33 \cdot 68$ & $1,1,0$ & $50 \cdot 47$ & $37 a$ & 8 & 0.00 & $90 \cdot 00$ & $18 \cdot 92$ & $1,0,0$ & 18.92 \\
\hline $13 a$ & 8 & 0.00 & $90 \cdot 00$ & $22 \cdot 62$ & $1,0,0$ & $22 \cdot 62$ & $37 b$ & 2 & $12 \cdot 53$ & $85 \cdot 35$ & $40 \cdot 60$ & $3,1,0$ & $43 \cdot 14$ \\
\hline $13 b$ & 6 & $18 \cdot 43$ & 76.66 & $71 \cdot 57$ & $1,1,1$ & $27 \cdot 79$ & $37 c$ & 6 & 36.87 & $71 \cdot 08$ & $53 \cdot 13$ & $1,1,1$ & $50 \cdot 57$ \\
\hline 15 & 2 & $19 \cdot 65$ & $82 \cdot 33$ & $42 \cdot 27$ & $2,1,0$ & $48 \cdot 19$ & $39 a$ & 6 & $21 \cdot 80$ & $75 \cdot 14$ & $68 \cdot 20$ & $1,1,1$ & $32 \cdot 20$ \\
\hline $17 a$ & 8 & 0.00 & $90 \cdot 00$ & 28.07 & $1,0,0$ & $28 \cdot 07$ & $39 b$ & 1 & $29 \cdot 20$ & 87.06 & $48 \cdot 12$ & $3,2,1$ & $50 \cdot 13$ \\
\hline $17 b$ & 4 & $45 \cdot 00$ & $86 \cdot 63$ & $45 \cdot 00$ & $2,2,1$ & 61.92 & $41 a$ & 8 & 0.00 & $90 \cdot 00$ & $12 \cdot 68$ & $1,0,0$ & $12 \cdot 68$ \\
\hline $19 a$ & 4 & $18 \cdot 44$ & 86.98 & $18 \cdot 44$ & $1,1,0$ & $26 \cdot 53$ & $41 b$ & 2 & $17 \cdot 10$ & $84 \cdot 40$ & 36.03 & $2,1,0$ & $40 \cdot 88$ \\
\hline $19 b$ & 6 & 33.69 & $71 \cdot 59$ & $56 \cdot 31$ & $1,1,1$ & $46 \cdot 83$ & $41 c$ & 4 & 36.87 & $77 \cdot 32$ & $36 \cdot 87$ & $1,1,0$ & $55 \cdot 88$ \\
\hline $21 a$ & 6 & 14.03 & $79 \cdot 02$ & 75.97 & $1,1,1$ & $21 \cdot 78$ & $43 a$ & 6 & $9 \cdot 46$ & 81.98 & $80 \cdot 54$ & $1,1,1$ & $15 \cdot 18$ \\
\hline $21 b$ & 2 & $22 \cdot 83$ & $79 \cdot 02$ & $50 \cdot 91$ & $2,1,1$ & $44 \cdot 41$ & $43 b$ & 2 & $12 \cdot 10$ & $87 \cdot 33$ & $24 \cdot 78$ & $2,1,0$ & 27.91 \\
\hline 23 & 2 & $15 \cdot 25$ & $82 \cdot 51$ & $52 \cdot 13$ & $3,1,1$ & $40 \cdot 45$ & $43 c$ & 4 & $45 \cdot 00$ & $80 \cdot 63$ & $45 \cdot 00$ & $3,3,2$ & $60 \cdot 77$ \\
\hline $25 a$ & 8 & 0.00 & $90 \cdot 00$ & $16 \cdot 26$ & $1,0,0$ & $16 \cdot 26$ & $45 a$ & 2 & $10 \cdot 30$ & $83 \cdot 62$ & 63.44 & $3,1,1$ & $28 \cdot 62$ \\
\hline $25 b$ & 2 & 36.87 & $90 \cdot 00$ & 36.87 & $3,3,1$ & $51 \cdot 68$ & $45 b$ & 2 & $26 \cdot 57$ & 83.62 & 63.43 & $2,2,1$ & 36.87 \\
\hline $27 a$ & 4 & $21 \cdot 80$ & $85 \cdot 75$ & $21 \cdot 80$ & $1,1,0$ & 31.59 & $45 c$ & 2 & $38 \cdot 66$ & 84.90 & $51 \cdot 34$ & $2,2,1$ & $53 \cdot 13$ \\
\hline $27 b$ & 2 & 15.07 & 85.75 & $31 \cdot 33$ & $2,1,0$ & $35 \cdot 43$ & $47 a$ & 2 & $26 \cdot 56$ & $87 \cdot 56$ & 63.44 & $3,3,1$ & 37.07 \\
\hline $29 a$ & 8 & 0.00 & $90 \cdot 00$ & $43 \cdot 60$ & $1,0,0$ & $43 \cdot 60$ & $47 b$ & 2 & $22 \cdot 71$ & $82 \cdot 67$ & $35 \cdot 39$ & $3,2,0$ & 43.66 \\
\hline $29 b$ & 2 & 33.69 & 84.06 & $56 \cdot 31$ & $2,2,1$ & $46 \cdot 40$ & $49 a$ & 6 & 30.96 & $72 \cdot 17$ & 59.04 & $1,1,1$ & $43 \cdot 57$ \\
\hline $31 a$ & 6 & $11 \cdot 31$ & $80 \cdot 72$ & $78 \cdot 69$ & $1,1,1$ & $17 \cdot 90$ & $49 b$ & 2 & $10 \cdot 62$ & $85 \cdot 32$ & $47 \cdot 49$ & $5,1,1$ & 43.57 \\
\hline $31 b$ & 2 & $27 \cdot 41$ & $78 \cdot 84$ & 43.66 & $2,1,1$ & $52 \cdot 20$ & $49 c$ & 2 & $30 \cdot 35$ & $75 \cdot 82$ & $49 \cdot 27$ & $3,2,2$ & $49 \cdot 23$ \\
\hline $33 a$ & 4 & 14.04 & $88 \cdot 26$ & $14 \cdot 04$ & $1,1,0$ & $20 \cdot 05$ & & & & & & & \\
\hline
\end{tabular}

Table 2. Classification of multiplicity $m$ for all cubic-cubic misorientations using quaternion representation

$\begin{array}{rcc}m & a & b \\ 48 & 1 & 0 \\ 16 & 1 & \sqrt{2}-1 \\ 12 & 1 & 1 / 3 \\ 8 & 1 & \sqrt{2}-1 \\ & 1 & b \\ 6 & 1 & b \\ 4 & 1 & b \\ & 1 & b \\ & 1 & b \\ 2 & 1 & b \\ & 1 & b \\ & 1 & b \\ & 1 & b \\ & 1 & \sqrt{2}-1 \\ 1 & \text { All others } & \end{array}$

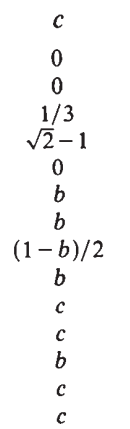

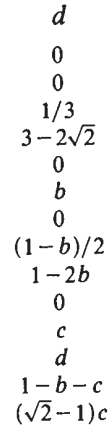

$(\sqrt{2}-1) c$
Conditions

$$
\begin{gathered}
\sqrt{2}-1>b>0 . \\
1 / 3>b>0 . \\
\sqrt{2}-1 \geq b>0 .
\end{gathered}
$$

$\sqrt{2}-1 \geq b>1 / 3$.

$\sqrt{2}-1>b>1 / 3$.

$\sqrt{2}-1 \geq b>c>0$

$\sqrt{2}-1 \geq b>c>0,1>b+2 c$.

$\sqrt{2}-1 \geq b>d>0,1>2 b+d$

$\sqrt{2}-1 \geq b>c>1-b-c$.

$\sqrt{2}-1>c>0$.
$\Sigma 29 b, 35 b, 45 b, 45 c, 47 a$ should be classified as $m=2$ because they lie upon the $A B D$ plane (but not upon the edges) of Fig. 4 in the paper. Boundaries $\Sigma 23,27 b, 35 a$, $37 b, 47 b, 49 b$ were erroneously classified as $m=1$ because the authors had difficulties in analyzing points on the curved surface $A B D$. It is confirmed now that every misorientation point on the surface $A C D$ should be classified as $m=2$; the inverse $g^{-1}$ for all points on this curved surface can be shown to be equivalent to $g$ under relation (44) of the paper. For the convenience of future usage, we have produced two tables in the following which classify the multiplicities $m$ of all cubic-cubic misorientations in Euler angle and quaternion representations. A cubic-cubic misorientation corresponds to an interior point of an asymmetric domain or one or two points on its surface. The multiplicity $m$ of a misorientation satisfies $m=1$ if the misorientation is represented by an interior point and $m \geq 1$ otherwise.

For quaternions $[a, b, c, d]$, the following is chosen as the definition of an asymmetric domain:

$$
b \geq c \geq d \geq 0, \quad a \geq(\sqrt{2}+1) b, \quad a \geq b+c+d
$$

Using normalization $a=1$, instead of the usual $a^{2}+b^{2}+$ $c^{2}+d^{2}=1$, we have classified all points of multiplicity in Table 2 .

In Fig. 1, multiplicities associated with points are indicated at the locations of the points and those associated with lines and planes are indicated by an oval and by a cross, respectively. For example, point $A$ is represented by quaternion $[1,0,0,0]$ and has multiplicity 48 ; line $B E$ is represented by quaternions of the form $[1, \sqrt{2}-1, c,(\sqrt{2}-$ 1)c] and associated with multiplicity 2. Plane $E B F$ is described by quaternions of the form $[1, b, b, d]$ and associated with multiplicity 2 . Points on the surface $B C E F$ related as $[1, \sqrt{2}-1, c, d]$ and $[1, \sqrt{2}-1,(c+d) / \sqrt{2}$, $(c-d) / \sqrt{2}]$ represent physically equivalent misorientations; all other points on the surface of the asymmetric domain represent distinct misorientations. Notice that $m=1$ if $c>d>0$ and $d \neq(\sqrt{2}-1) c$ in $[1, \sqrt{2}-1, c, d]$.

For Euler angle representation, an asymmetric domain is defined by the following relations: $0 \leq \cos \phi \leq$ $\sin \varphi_{1} \sin \varphi_{2} /\left(1+\cos \varphi_{1} \cos \varphi_{2}\right)$ and $0 \leq \varphi_{1} \leq \varphi_{2} \leq \pi / 2-\varphi_{1}$, $\arccos (1 / 3) \leq \phi \leq \pi / 2$. Table 3 gives all multiplicities in this space. 
Table 3. Classification of multiplicity $m$ for all cubic-cubic misorientations using Euler angles

$\begin{array}{rcccc}m & \varphi_{1} & \phi & \varphi_{2} & \text { Conditions and comments } \\ 48 & 0 & \pi / 2 & 0 & \\ & 0 & \pi / 2 & \pi / 2 & (0, \pi / 2,0) \text { and }(0, \pi / 2, \pi / 2) \text { are equivalent } \\ 16 & 0 & \pi / 2 & \pi / 4 & \\ 12 & \pi / 4 & \arccos (1 / 3) & \pi / 4 & \\ 8 & \pi / 4 & \pi / 2 & \pi / 4 & 0<\varphi_{2}<\pi / 2, \varphi_{2} \neq \pi / 4 \\ & 0 & \pi / 2 & \varphi_{2} & \cos \phi=\sin 2 \varphi /\left(2+\sin _{2} \varphi\right), 0<\varphi<\pi / 4 \\ 6 & \varphi & \phi & \pi / 2-\varphi & \cos \phi=\sin ^{2} \varphi /\left(1+\cos ^{2} \varphi\right), 0<\varphi<\pi / 4, \\ 4 & \varphi & \phi & \arccos (1 / 3)<\phi<\pi / 2 \\ & \pi / 4 & \phi & \varphi_{2} & \cos \phi=\sin \varphi_{1} \sin \varphi_{2} /\left(1+\cos \varphi_{1} \cos \varphi_{2}\right) \\ 2 & \varphi_{1} & \phi & 0<\varphi_{1}<\varphi_{2}<\pi / 2-\varphi_{1} \\ & \varphi_{1} & \pi / 2 & \pi / 4 & 0<\varphi_{1}<\pi / 4 \\ & \varphi & \phi & \varphi & 0<\varphi<\pi / 4,0 \leq \cos \phi<\sin { }^{2} \varphi /\left(1+\cos ^{2} \varphi\right) \\ & \varphi & \phi & \pi / 2-\varphi & 0<\varphi<\pi / 4,0 \leq \cos \phi<\sin 2 \varphi /\left(2+\sin ^{2} 2 \varphi\right)\end{array}$

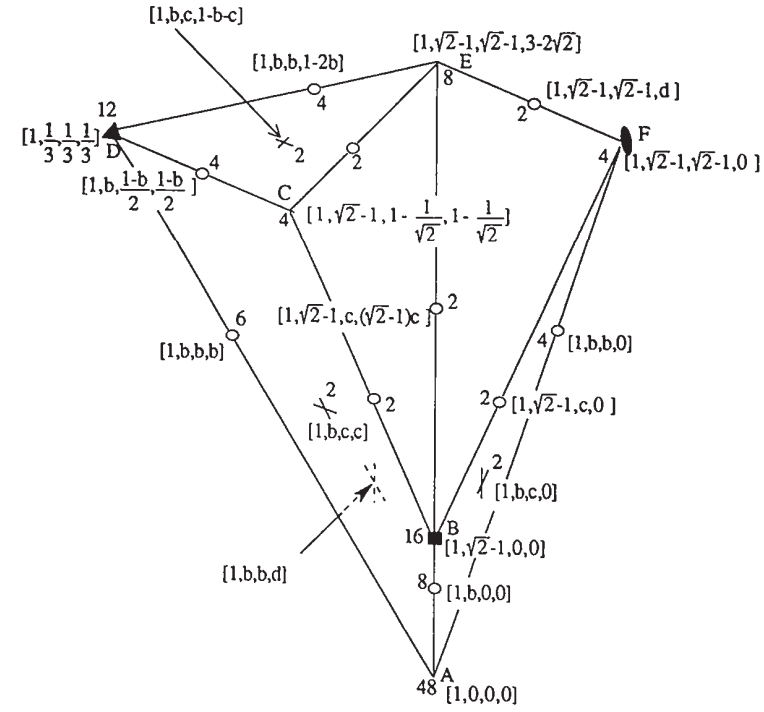

Fig. 1. Graphical representation of Table 2.

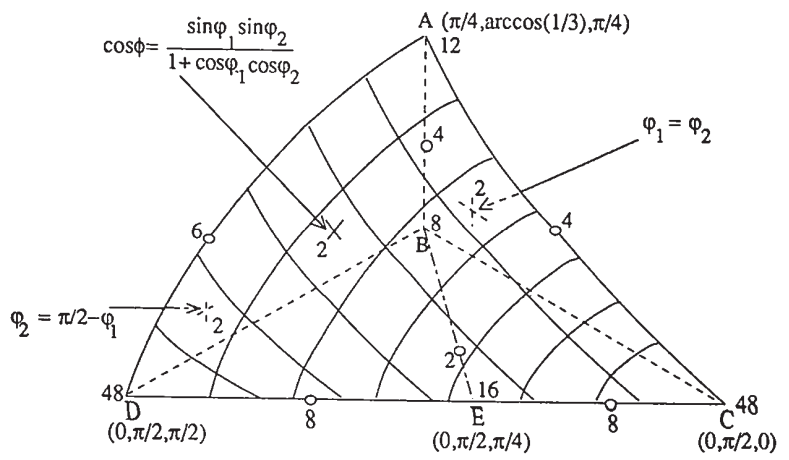

Fig. 2. Graphical representation of Table 3.
The content of Table 3 is illustrated in Fig. 2 which shows the surface of the asymmetric domain.

Again multiplicities associated with lines (curves) are indicated by ovals, and surfaces by crosses. For example, point $E$ has coordinates $(0, \pi / 2, \pi / 4)$ in the Euler space and possesses multiplicity 16 . Curve $A D$ has multiplicity 6 and the curved surface $A C D$ is associated with multiplicity 2 . Points on the surface $B C D$ related as $\left(\varphi_{1}, \pi / 2\right.$, $\left.\varphi_{2}\right)$ and $\left(\varphi_{1}, \pi / 2, \pi / 2-\varphi_{2}\right)$ represent physically equivalent misorientations; ${ }^{*}$ all other points on the surface of the asymmetric domain represent distinct misorientations. Notice that $m=1$ if $0<\varphi_{1}<\varphi_{2}<\pi / 2-\varphi_{1}$ and $\varphi_{2} \neq \pi / 4$ in $\left(\varphi_{1}, \pi / 2, \varphi_{2}\right)$.

BLA and JZ acknowledge the support of the Office of Basic Energy Science of the US Department of Energy under grant No. DE-FG02-88ER45355. Help from Stuart Wright with the figures is gratefully acknowledged.

* An example is $\Sigma 25 b$ which is represented by the point (36.87 $\left.90^{\circ}, 36.87^{\circ}\right)$ on $B C$ and by $\left(36.87^{\circ}, 90^{\circ}, 53 \cdot 13^{\circ}\right)$ on $B D$ of Fig. 2 .

\section{References}

Altmann, S. L. (1986). Rotations, Quaternions and Double Groups. Oxford: Clarendon Press.

Bunge, H. J. (1982). Texture Analysis in Materials Science. London: Butterworth.

FranK, F. C. (1988). Metall. Trans. A19, 403-408.

Gelfand, I. M., Minlos, R. A. \& Shapiro, Z. YA. (1963). Representations of the Rotation and Lorentz Groups and their Applications. Oxford: Pergamon.

Grimmer, H. (1989). Acta Cryst. A45, FC1-FC2.

HANDSCOMB, D. C. (1958). Can. J. Math. 10, 85-88.

Pospiech, J., SzTwiertniA, K. \& Haessner, F. (1986). Textures Microstruct. 6, 201-215.

ZhaO, J. \& ADAMS, B. L. (1988). Acta Cryst. A44, 326-336. 